\title{
Person Detection and Re-identification Across Multiple Images and Videos Obtained via Crowdsourcing
}

\author{
Yu Zheng, Zhenhua(Jimmy) Chen, Senem Velipasalar and Jian Tang \\ Department of Electrical Engineering and Computer Science \\ Syracuse University \\ Syracuse, NY 13244 \\ \{yzheng04, zchen03, svelipas, jtang02\} esyr.edu
}

\begin{abstract}
Person re-identification is indispensable for consistent labeling across different camera views. Most existing studies use static cameras, apply background subtraction to detect moving people, and then focus on the matching of detection results. However, if cameras are mobile or only single image frames (not videos) are available, then background subtraction cannot be used, and human detection needs to be performed on entire images. In this paper, different from most of the existing work, we focus on a crowdsourcing scenario to find and follow person(s) of interest in the collected images/videos. We propose a novel approach combining R-CNN based person detection with the GPU implementation of color histogram and SURFbased re-identification. Moreover, GeoTags are extracted from the EXIF data of videos captured by smart phones, and are displayed on a map together with the time-stamps. All the processing is performed on a GPU, and the average processing time is $5 \mathrm{~ms}$ per frame.
\end{abstract}

\section{INTRODUCTION}

Large camera networks are increasingly being deployed in public places such as airports, subways, campuses and office buildings. These cameras are usually installed across wider areas with non-overlapping fields of view (FOV) to provider better coverage. However, with these setups, tracking of person(s) is still limited to the areas where the cameras are installed. Thanks to the widespread use of smart phones, crowdsourcing of videos has the potential to provide a much larger coverage for longer periods of time. For instance, a video or image captured by someone at a remote location (with no pre-installed camera) can have the person of interest in it. If it is possible to have access to large number of videos captured at different locations over time, this would potentially allow following a person over a much larger area for extended periods of time. However, the extremely large amount of data captured in these scenarios makes it impossible for humans to perform manual analysis, and necessitates autonomous analysis of data. Video analysis enable long term characterization and modeling of the people in the scene. Such application is required for high-level surveillance tasks and make them even smarter [6].

Permission to make digital or hard copies of all or part of this work for personal or classroom use is granted without fee provided that copies are not made or distributed for profit or commercial advantage and that copies bear this notice and the full citation on the first page. Copyrights for components of this work owned by others than ACM must be honored. Abstracting with credit is permitted. To copy otherwise, or republish, to post on servers or to redistribute to lists, requires prior specific permission and/or a fee. Request permissions from permissions@acm.org.

ICDSC '16, September 12-15, 2016, Paris, France

(c) 2016 ACM. ISBN 978-1-4503-4786-0/16/09. . $\$ 15.00$

DOI: http://dx.doi.org/10.1145/2967413.2967421
Given an image/video of person of interest, re-identification is the process of identifying same person in the images/video captured by a different camera. Re-identification is indispensable in establishing the consistent labeling across multiple cameras or even within the same camera to connect broken trajectories or re-establish lost tracks. Person re-identification is a difficult problem due to large variations in person's appearance, lighting conditions and contrast across different cameras. Person re-identification/association across different camera views has two main parts; (1) detecting the people in the scene and (2) re-identifying them in other camera views. The face detection method proposed by Viola and Jones [27], and the human detection method, based on histograms of oriented gradients, proposed by Dalal and Triggs [8] are two of the important works on detection. Dollar et al. [9] proposed Integral Channel Features for pedestrian detection. Local Binary Patterns [29], multiple kernels [26] and part-based models [10] have also been introduced for human detection.

Deep Convolutional Neural Networks (CNNs) have received a lot of attention recently, especially after achieving very good performance in the ImageNet challenge [17]. Later, Girshick et al. [12] combined region proposals with CNNs, and introduced R-CNN, regions with $\mathrm{CNN}$ features, for object detection. Then, faster R$\mathrm{CNN}$ [24] has been proposed, which focus on the speed up by pruning the hypothesis in detection.

Person re-identification has been studied by many researchers in the past few years $[5,4,28,11,25,20,21,22]$. Earlier research focused on taking advantage of camera inner parameters during the matching process. Most of the existing work relies on the appearance-based similarity between images, such as color and texture of clothing, to establish correspondences. In general, recent approaches focus on three aspects; (1) designing subjectdiscriminative [30], descriptive and robust visual descriptors to characterize a person's appearance [1], (2) using feature transformation which projects features between different camera-dependent spaces, such as feature warping [19], and sparse basis expansion [18, $16]$, and (3) learning suitable distance metrics that maximize the chance of a correct matching [2, 14, 23].

In many of the existing works, cameras are static and background subtraction is applied to detect moving objects. This significantly simplifies and speeds up the detection stage. Then, focus is placed on the matching part. However, if cameras are mobile or only single images (not videos) are available, then background subtraction cannot be employed. In this case, human detection needs to be performed in the entire image, which is in general a computationally intensive process. In this paper, different from most of the existing work, we focus on a crowdsourcing scenario to find and follow person(s) of interest in the collected images/videos. We propose a novel approach combining R-CNN based person detection 
with the GPU implementation of color histogram and SURF-based re-identification. Moreover, the GeoTags are extracted from the EXIF data of videos captured by smart phones, and these locations are displayed on a map together with the time-stamps for a spatiotemporal representation/visualization of the trajectory. All the processing, including R-CNN based detection, histogram correlation and SURF-based matching, is performed on GPU. The average processing time for the proposed detection and matching algorithm is 5 ms per frame on an NVIDIA Quadro K5200 8GB GPU. The experimental results show the promise of the proposed method.

The rest of the paper is organized as follows: The proposed approach is described in detail in Sec. 2. The experimental results are presented in Sec. 3, and the paper is concluded in Sec. 4.

\section{PROPOSED METHOD}

In our proposed method, we use the pre-trained Region-based Convolutional Networks (R-CNN) [12], implemented with Caffe [15], to detect people in images. We refer to the image containing the person(s) of interest as the query image and the frames in the video(s) to be examined as the candidates images. The R-CNN detector, which is run on all video images, returns the bounding boxes of candidate people regions in each image. Then, both the color histogram and SURF features are extracted from the candidate regions to be matched with the subject(s) of interest. After a match is found, GeoTag information is extracted from the matched video to generate the spatio-temporal model for candidates, and mark their path on a map. The overall flow diagram of the proposed method is provided in Fig. 1.

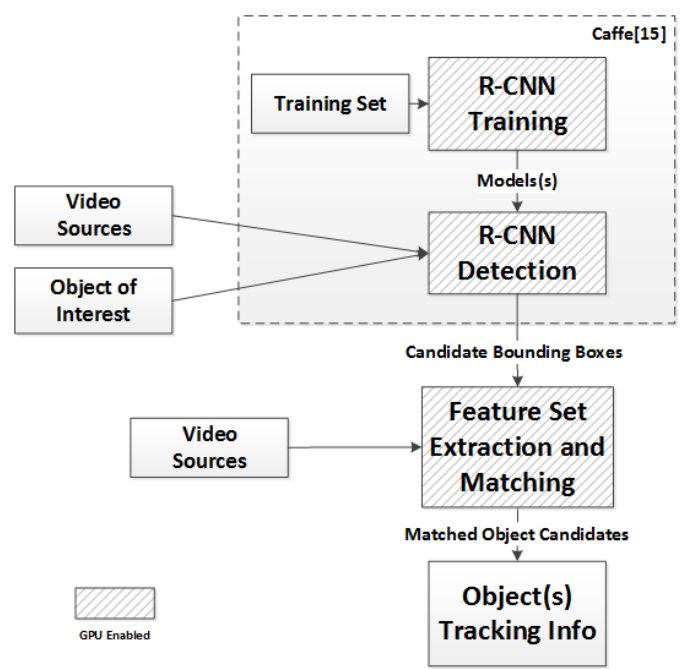

Figure 1: Flow diagram of the proposed method.

\subsection{R-CNN-based Detection}

We apply the R-CNN model [12] provided in Caffe for better performance in detection results. A higher detection rate will provide better matching results across different views. In our experiments, we observed that all people in the videos were successfully detected by the R-CNN detector.

The architecture of the used R-CNN is shown in Fig. 2. It has 7 layers. Since the training images for the convolutional neural network needs to be a fixed-size of $227 \times 227$ pixels, all the images are resized to the required size first. As stated in [7], in the first layer, the resized images are convolved with 96 kernels of size $11 \times 11 \times 3$ pixels with a stride of 4 pixel, and then max-pooling is applied in $3 \times 3$ grid. The second layer has the same framework, with 256 kernels of size $5 \times 5 \times 48$. Layer 3 and 4 are two convolution layers without pooling, which both have 384 kernels. Layer 5 is similar to layer 2 . Layer 6 and 7 are fully connected layers with 4096 nodes. The activation function used in convolution and fully connected layer is Rectified Linear function. More details can be found in [17] and [12].

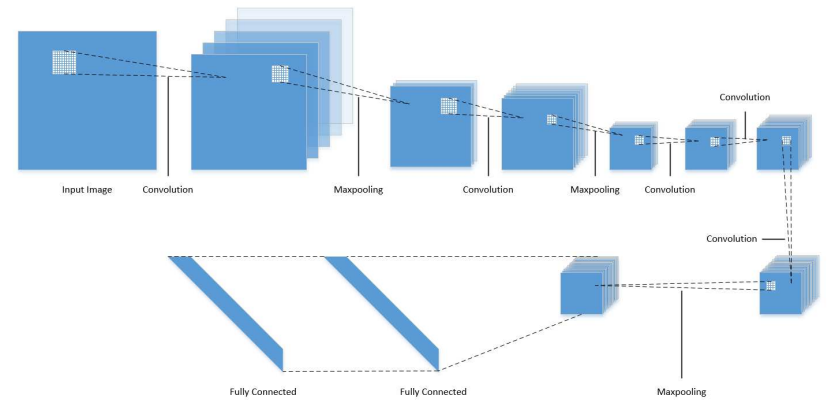

Figure 2: Architecture of used R-CNN model.

\subsection{GPU Accelerated Re-identification}

The process of detection and re-identification of people across many different views can be computationally very expensive. Each region proposed by the $\mathrm{R}-\mathrm{CNN}$ on each candidate image has to be compared with the representation of person(s) of interest in the query image to see if there is a match. In order to reduce the processing time of each video frame, we implemented a GPU-based parallel matching strategy, which speeds up the detection and reidentification phase. The matching procedure has the following steps:

1. Apply R-CNN based detection on video frames to get bounding boxes for candidate people regions.

2. Convert candidate regions to HSV color space, compute histograms and compare with the histogram of the person(s) of interest from the query image by using color correlation.

3. If the histogram correlation score is higher than a threshold $\tau$, apply SURF to detect matching feature points between candidate regions and images person(s) of interest.

As mentioned previously, R-CNN detection provides the coordinates of the bounding box around candidate people regions. Then, we convert this region to the HSV color space to reduce the effect of illumination changes. Afterwards, we calculate the color histograms, and compare the histograms by using color correlation calculation in (1), where $H_{1}$ and $H_{2}$ are the two color histograms, $N$ is the total number of histogram bins, and $\bar{H}=\frac{1}{N} \sum_{i} H(i)$.

$$
d\left(H_{1}, H_{2}\right)=\frac{\sum_{i=1}^{N}\left(H_{1}(i)-\overline{H_{1}}\right)\left(H_{2}(i)-\overline{H_{2}}\right)}{\sqrt{\sum_{i=1}^{N}\left(H_{1}(i)-\overline{H_{1}}\right)^{2}\left(H_{2}(i)-\overline{H_{2}}\right)^{2}}} .
$$

If the histogram correlation score is higher than a threshold $\tau$, a matcher based on Speeded Up Robust Features (SURF) [3] is applied to detect matching feature points between candidate regions and images person(s) of interest. SURF is a fast, scaleand rotation-invariant detector and descriptor, which outperforms previous methods with respect to repeatability, distinctiveness and robustness. In this work, a SURF-based detector and matcher is implemented on GPU to recognize the same person from multiple different views. If the number of matched points is larger than $50 \%$ of the smaller number of points (between the candidate region and the person of interest), a match is declared. 


\subsection{Spatio-Temporal Model}

The prevalence of smart phones not only provides large volumes of video/image data with ever-increasing quality, but also makes GPS information, via GeoTags, more accessible. To generate the spatio-temporal model of a person, who is matched across different videos captured by different phones at different locations, we extract the EXIF information, and then visualize it on GOOGLEMAP API. EXIF is an exchangeable file format that contains the metadata embedded within the video. As shown in Fig 3, it includes information such as the length of the video, GPS location, timestamp and compression rate. We use EXIFtool [13] to extract the GeoTags, and obtain the location where the video was initially captured.

It should be noted that the GeoTags only provide the GPS location of the device when it starts recording the video. Thus, we need to estimate the distance between the target and the device to display the location of the target. We do this by using the bounding box sizes for people obtained at four different pre-defined distances from smart phones. Table 1 provides the list of bounding box sizes obtained at $20 \mathrm{ft}, 40 \mathrm{ft}, 60 \mathrm{ft}$ and $80 \mathrm{ft}$ from the capturing smart phone. We find the closest bounding box size from this list and use the corresponding distance as the distance of the target from the phone. Then, we draw a circle with this radius around the GPS location of the device. Since the videos are obtained through crowdsourcing, there can be another video of the same person captured around the same time. We follow the same steps for the location from another device, and use the intersection of the circles as the location of the target. This process is illustrated in Fig. 4.

As mentioned above, the GPS location is only available for the first frame of the video. Thus, to be able to track the location of a target over time, we need many videos captured at different times, and this is when crowdsourcing comes to the rescue. When there are enough videos obtained through crowdsourcing, it will provide a more complete spatio-temporal picture of the trajectory of the target. It should be noted that, the proposed approach can be applied to images as well as videos. Since, each image has its own GPS and time-stamp information, localization will be more precise compared to videos. To be able to use the intersection of the circles as the location, when we have videos as the input, we used short videos in our experiments (since GPS data is only available for the first frame).

\begin{tabular}{|c|c|}
\hline Distance from camera (ft) & Bounding box size \\
\hline 20 & $300 \times 950$ \\
\hline 40 & $143 \times 454$ \\
\hline 60 & $100 \times 283$ \\
\hline 80 & $68 \times 220$ \\
\hline
\end{tabular}

Table 1: Bounding box sizes for different distances from the camera.

\section{EXPERIMENTAL RESULTS}

As mentioned above, all the processing, including R-CNN based detection, histogram correlation and SURF-based matching, is performed on GPU. The average processing time for the proposed detection and matching algorithm is $5 \mathrm{~ms}$ per frame on an NVIDIA Quadro K5200 8GB GPU.

We performed different experiments using videos captured by multiple smart phones at different places. The targets are seen from different views (frontal, side view etc.) in the captured videos. In the first experiment, a total of three videos were recorded, from three different phones, capturing a target from different angles, namely front view, side view and back view. Figure 5 shows the

\begin{tabular}{|c|c|}
\hline Create Date & $\begin{array}{l}\text { 2016:05:05 } 15: 46: 29 \\
5 \text { days, } 21 \text { hours, } 17 \text { minutes, } 6 \text { seconds ago }\end{array}$ \\
\hline Modify Date & $\begin{array}{l}\text { 2016:05:05 } 15: 46: 40 \\
5 \text { days, } 21 \text { hours, } 16 \text { minutes, } 55 \text { seconds ago }\end{array}$ \\
\hline Time Scale & 600 \\
\hline Preferred Rate & 1 \\
\hline Source Image Width & 1,920 \\
\hline Source Image Height & 1,080 \\
\hline Compressor Name & H. 264 \\
\hline Bit Depth & 24 \\
\hline Video Frame Rate & 29.981 \\
\hline Avg Bitrate & $17.3 \mathrm{Mbps}$ \\
\hline GPS Altitude & $172 \mathrm{~m}$ \\
\hline GPS Altitude Ref & Above Sea Level \\
\hline GPS Latitude & 43.037800 degrees $\mathrm{N}$ \\
\hline GPS Longitude & 76.131500 degrees $W$ \\
\hline GPS Position & 43.037800 degrees $N, 76.131500$ degrees $W$ \\
\hline Megapixels & 2.1 \\
\hline Rotation & 0 \\
\hline
\end{tabular}

Figure 3: Exif information from video

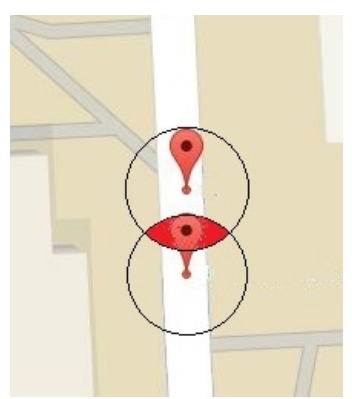

Figure 4: Determining the location of a target.

query target image and the matched images, from different cameras, showing the target from different angles. The color histogram correlation scores are shown in Table 2. As expected, since the query is a back view, the correlation between the query and a candidate region with the back view is the highest. The correlation score for the front view comes next, and the score between the query and a side view is the smallest for the same person. This experiment also allowed us to determine an empirical threshold for $\tau$ that allows matching the same person from different views, and also having a discriminative power for different people. The GeoTag locations on a map, for this experiment, are displayed in Fig. 6.

The second experiment scenario has a more complicated setup involving the detection and matching of three different targets simultaneously. There are four different smart phones capturing videos of these three targets from different locations and angles. To better simulate a crowdsourcing scenario, a total of 38 videos are recorded from these four devices so that there are more videos to process, which would provide a more complete GeoTag information over time. Each video contains at least one target or more.

Example matching images are shown in Fig. 8. There is a large variation in the distance of targets from the capturing device, which can be observed from the original frames as well as the lower resolution of some of the detected targets. All three candidates have been successfully re-identified across different cameras, capturing from different angles, by histogram correlation and SURF-based 
matching. Table 3 shows example color correlation scores between the query image and all three candidates. As can be seen, the highest correlation scores are across the diagonal. Fig. 7 shows the GeoTag locations on a map for all three targets over time. Red, green and yellow marks correspond to targets one, two and three, respectively.

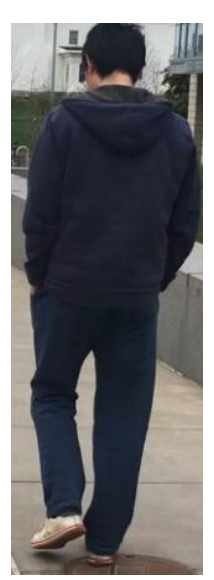

(a)

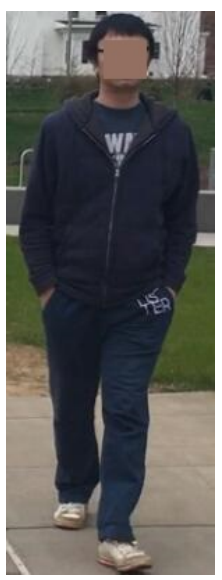

(b)

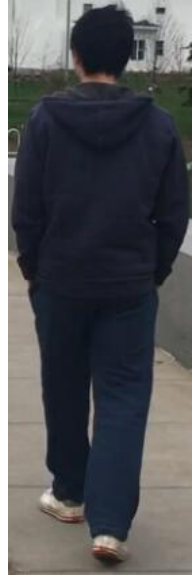

(c)

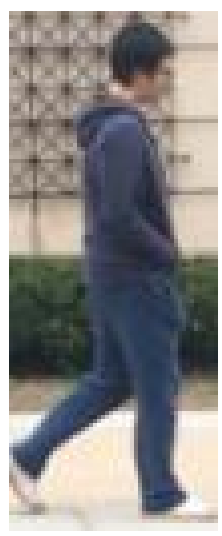

(d)
Figure 5: Detection and matching results to the query image in (a). Matched candidates from (b) front view, (c) back view, and (d) side view.

\begin{tabular}{|c|c|}
\hline Experiment 1 & Correlation score \\
\hline Front view & 0.6875 \\
\hline Back view & 0.7124 \\
\hline Side view & 0.4992 \\
\hline
\end{tabular}

Table 2: Color histogram correlation scores obtained between back, and front and side views in the first experiment.

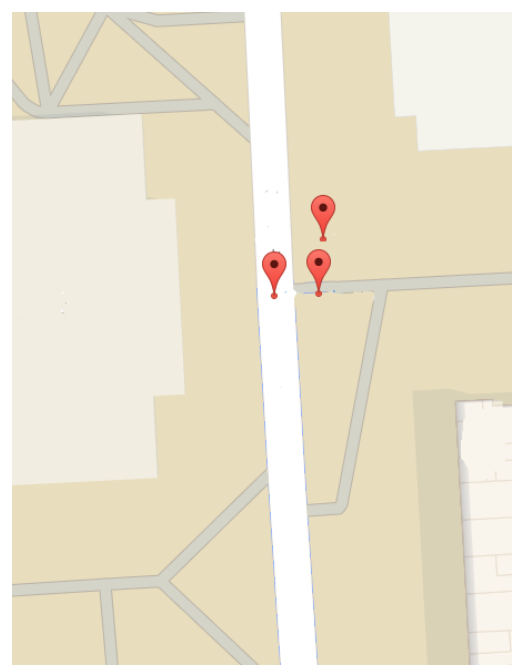

Figure 6: The GeoTag locations displayed on a map for the first experiment.

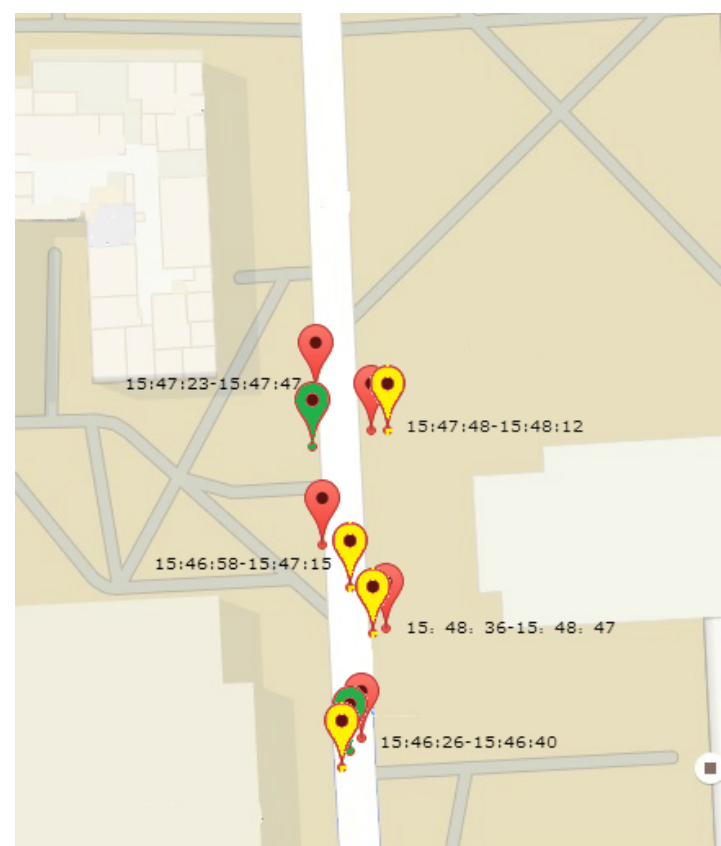

Figure 7: GeoTag results on the map for the second experiment.

\begin{tabular}{|c|c|c|c|}
\hline Color correlation & candidate 1 & candidate 2 & candidate 3 \\
\hline Query 1 & 0.6847 & 0.3975 & 0.1875 \\
\hline Query 2 & 0.4434 & 0.6685 & 0.2349 \\
\hline Query 3 & 0.1524 & 0.2569 & 0.7176 \\
\hline
\end{tabular}

Table 3: Color histogram correlation scores between the query and candidate regions for the second experiment.

\section{CONCLUSION}

We have proposed a method, which performs R-CNN based person detection and then person re-identification via matching on GPU. A pre-trained model from R-CNN is used to detect person candidate regions in videos. Then, both color and SURF features are extracted for each candidates, and used for matching with the person of interest. The GPS location in the image/video EXIF information is used to obtain a spatio-temporal model for the path taken by the target, and these locations are displayed on a map. All the processing is performed on GPU, and takes an average of $5 \mathrm{~ms}$ to process one frame. As feature work, we will incorporate more features to improve the re-identification.

\section{ACKNOWLEDGMENTS}

This work has been funded in part by Nation Science Foundation (NSF) under CAREER grant CNS-1206291, NSF grants CNS130255 and CNS-1525920.

\section{REFERENCES}

[1] Z. Akhtar, N. Martinel, C. Micheloni, and G. L. Foresti. Mobile re-identification based on local features analysis. In Proceedings of the International Conference on Distributed Smart Cameras, ICDSC '14, pages 11:1-11:6, New York, NY, USA, 2014. ACM.

[2] S. Bąk, G. Charpiat, E. Corvée, F. Brémond, and M. Thonnat. Learning to Match Appearances by Correlations in a Covariance Metric Space, pages 806-820. Springer Berlin Heidelberg, Berlin, Heidelberg, 2012. 
[3] H. Bay, A. Ess, T. Tuytelaars, and L. Van Gool. Speeded-up robust features (SURF). Comput. Vis. Image Underst., 110(3):346-359, June 2008.

[4] L. Bazzani, M. Cristani, and V. Murino. Symmetry-driven accumulation of local features for human characterization and re-identification. Computer Vision and Image Understanding, 117(2):130-144, 2013. cited By 39.

[5] L. Bazzani, M. Cristani, A. Perina, M. Farenzena, and V. Murino. Multiple-shot person re-identification by he signature. pages 1413-1416, 2010. cited By 43.

[6] A. Bedagkar-Gala and S. K. Shah. A survey of approaches and trends in person re-identification. Image and Vision Computing, 32(4):270 - 286, 2014.

[7] X. Chen, P. Wei, W. Ke, Q. Ye, and J. Jiao. Pedestrian detection with deep convolutional neural network. In Computer Vision - ACCV 2014 Workshops, volume 9008 of Lecture Notes in Computer Science, pages 354-365. Springer International Publishing, 2015.

[8] N. Dalal and B. Triggs. Histograms of oriented gradients for human detection. In Proceedings of the 2005 IEEE Computer Society Conference on Computer Vision and Pattern Recognition (CVPR'05) - Volume 1 - Volume 01, CVPR '05, pages 886-893, Washington, DC, USA, 2005. IEEE Computer Society.

[9] P. Dollár, Z. Tu, P. Perona, and S. Belongie. Integral channel features. In British Machine Vision Conference (BMVC), London, England, 2009.

[10] P. F. Felzenszwalb, R. B. Girshick, D. McAllester, and D. Ramanan. Object detection with discriminatively trained part-based models. IEEE Trans. Pattern Anal. Mach. Intell., 32(9):1627-1645, Sept. 2010.

[11] N. Gheissari, T. Sebastian, and R. Hartley. Person reidentification using spatiotemporal appearance. In Computer Vision and Pattern Recognition, 2006 IEEE Computer Society Conference on, volume 2, pages 1528-1535, 2006.

[12] R. Girshick, J. Donahue, T. Darrell, and J. Malik. Rich feature hierarchies for accurate object detection and semantic segmentation. In Proceedings of the IEEE Conference on Computer Vision and Pattern Recognition (CVPR), 2014.

[13] P. Harvey. ExifTool by Phil Harvey. http://www.sno.phy.queensu.ca/ phil/exiftool/, 2008. [Online; accessed 19-April-2016].

[14] M. Hirzer, P. M. Roth, M. Koestinger, and H. Bischof. Relaxed pairwise learned metric for person re-identification. In Proc. European Conference on Computer Vision (ECCV), 2012.

[15] Y. Jia, E. Shelhamer, J. Donahue, S. Karayev, J. Long, R. Girshick, S. Guadarrama, and T. Darrell. Caffe: Convolutional architecture for fast feature embedding. arXiv preprint arXiv: 1408.5093, 2014.

[16] S. Karanam, Y. Li, and R. J. Radke. Sparse re-id: Block sparsity for person re-identification. In 2015 IEEE Conference on Computer Vision and Pattern Recognition Workshops (CVPRW), pages 33-40, June 2015.

[17] A. Krizhevsky, I. Sutskever, and G. E. Hinton. Imagenet classification with deep convolutional neural networks. In F. Pereira, C. Burges, L. Bottou, and K. Weinberger, editors, Advances in Neural Information Processing Systems 25, pages 1097-1105. Curran Associates, Inc., 2012.

[18] G. Lisanti, I. Masi, A. D. Bagdanov, and A. D. Bimbo. Person re-identification by iterative re-weighted sparse ranking. IEEE Transactions on Pattern Analysis and Machine Intelligence, 37(8):1629-1642, Aug 2015.

[19] N. Martinel, A. Das, C. Micheloni, and A. K. Roy-Chowdhury. Re-identification in the function space of feature warps. IEEE Transactions on Pattern Analysis and Machine Intelligence, 37(8):1656-1669, Aug 2015.

[20] N. Martinel, G. L. Foresti, and C. Micheloni. Person reidentification in a distributed camera network framework. IEEE Transactions on Cybernetics, PP(99):1-12, 2016.

[21] N. Martinel and C. Micheloni. Classification of local eigen-dissimilarities for person re-identification. IEEE Signal Processing Letters, 22(4):455-459, April 2015.

[22] N. Martinel, C. Micheloni, and G. L. Foresti. Kernelized saliency-based person re-identification through multiple metric learning. IEEE Transactions on Image Processing, 24(12):5645-5658, Dec 2015.

[23] A. Mignon and F. Jurie. Pcca: A new approach for distance learning from sparse pairwise constraints. In Computer Vision and Pattern Recognition (CVPR), 2012 IEEE Conference on, pages 2666-2672, June 2012.

[24] S. Ren, K. He, R. Girshick, and J. Sun. Faster R-CNN: Towards real-time object detection with region proposal networks. In Neural Information Processing Systems (NIPS), 2015.

[25] R. Satta. Appearance descriptors for person re-identification: a comprehensive review. CoRR, abs/1307.5748, 2013.

[26] A. Vedaldi, V. Gulshan, M. Varma, and A. Zisserman. Multiple kernels for object detection. In Proceedings of the International Conference on Computer Vision (ICCV), 2009.

[27] P. Viola and M. J. Jones. Robust real-time face detection. Int. J. Comput. Vision, 57(2):137-154, May 2004.

[28] X. Wang, G. Doretto, T. Sebastian, J. Rittscher, and P. Tu. Shape and appearance context modeling. In Computer Vision, 2007. ICCV 2007. IEEE 11th International Conference on, pages 1-8, Oct 2007.

[29] X. Wang, T. Han, and S. Yan. An hog-lbp human detector with partial occlusion handling. In Computer Vision, IEEE 12th International Conference on, pages 32-39, Sept 2009.

[30] Z. Wu, Y. Li, and R. J. Radke. Viewpoint invariant human re-identification in camera networks using pose priors and subject-discriminative features. IEEE Transactions on Pattern Analysis and Machine Intelligence, 37(5):1095-1108, May 2015. 


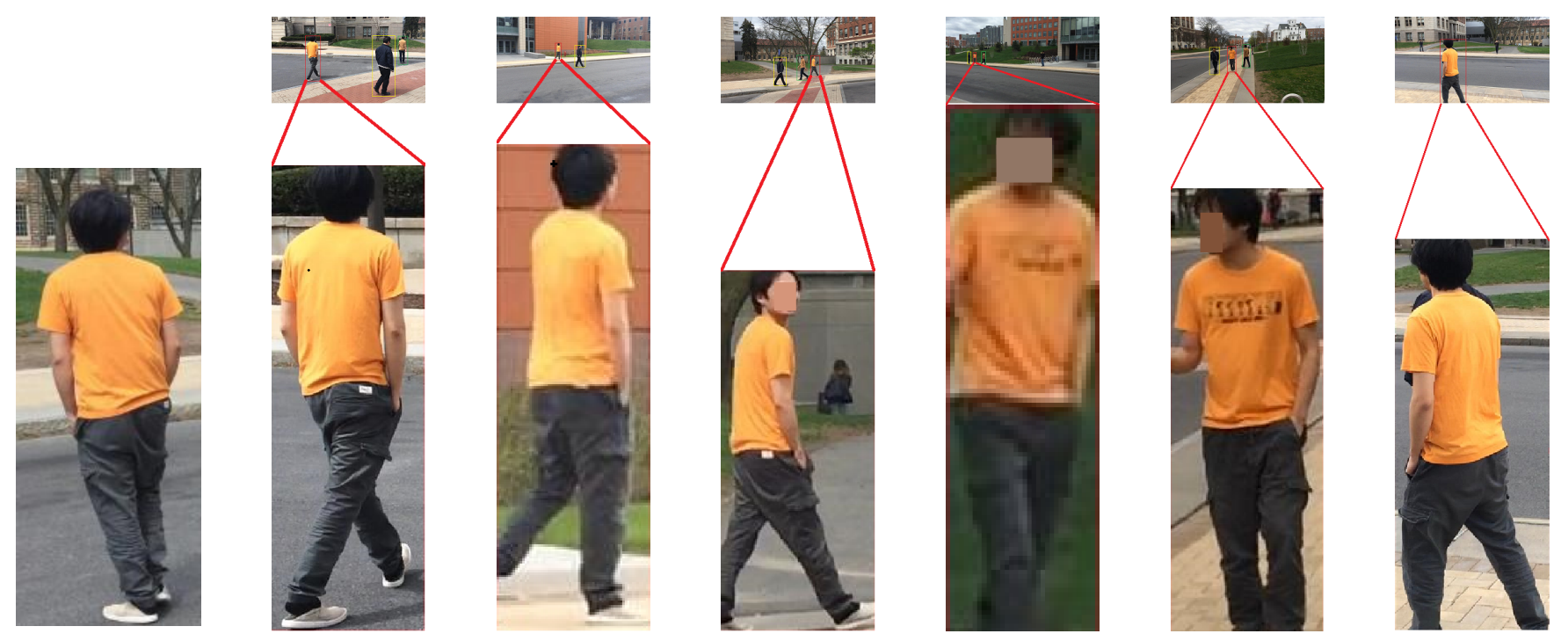

(a) Query image for the first target
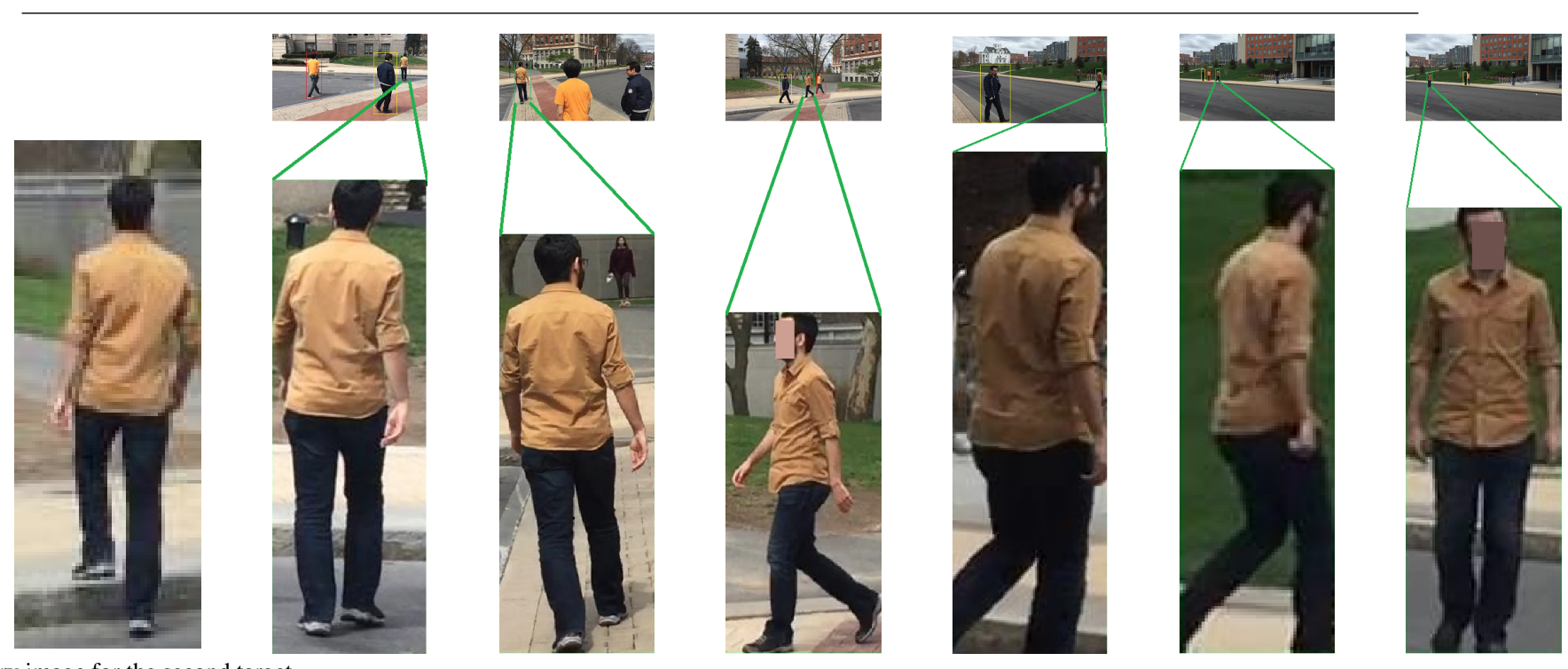

(b) Query image for the second target
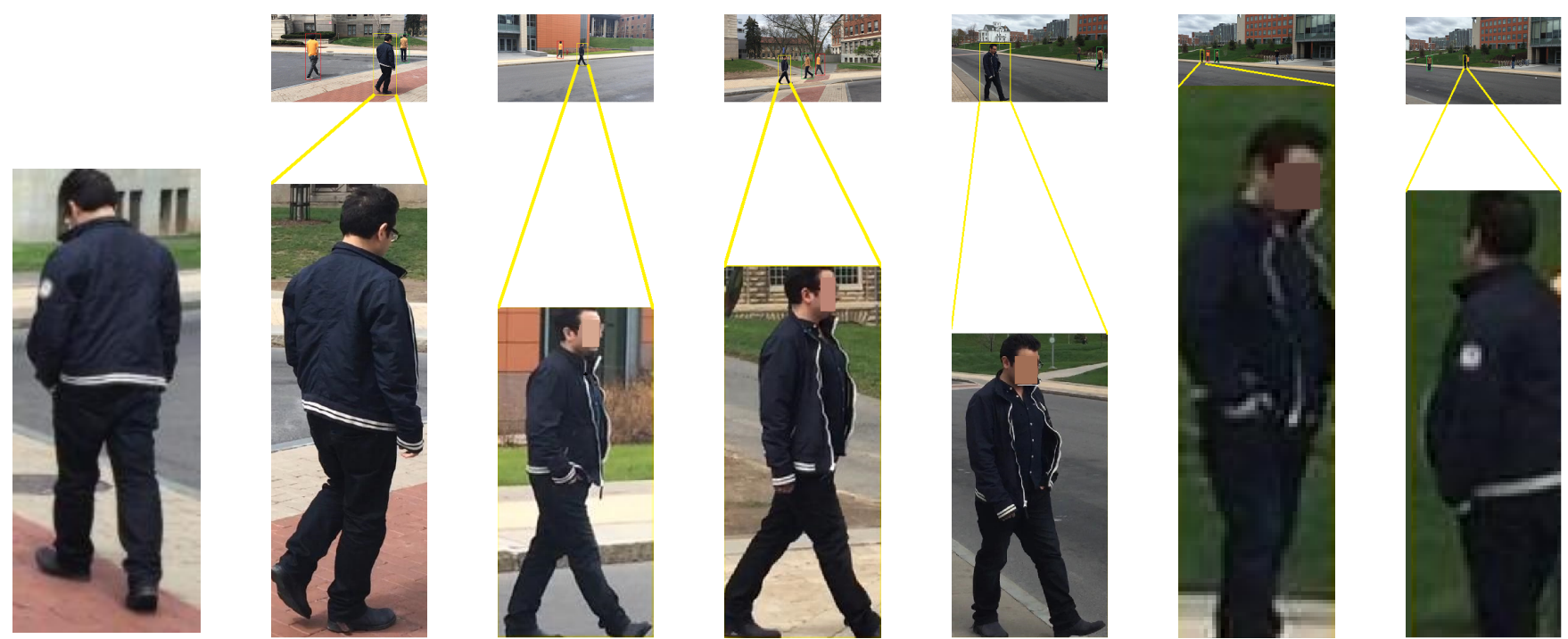

(c) Query image for the third target

Figure 8: Matching results for the experiment involving the detection and matching of three different targets simultaneously. 